\title{
Análisis de la soldadura aplicada en el ZSB Trager VW SUV Tiguan (soporte zsb)
}

\section{Analysis of the welding applied in the ZSB Trager VW SUV Tiguan (zsb support)}

\author{
MEDINA-MENDOZA, José Antonio†*, HERRERA-REYES, Naieli y MÉNDEZ-MACÍAS, Gerardo \\ Universidad Tecnológica del Norte de Aguascalientes, Departamento de Ingenierías, Aguascalientes, México.
}

ID $1^{\text {er }}$ Autor: José Antonio, Medina-Mendoza / ORC ID: 0000-0002-3969-5765, CVU CONACYT ID: 522777

ID $1^{\text {er }}$ Coautor: Naieli, Herrera-Reyes / ORC ID: 0000-0002-7654-1167, CVU CONACYT ID: 564450

ID $2^{\text {do }}$ Coautor: Gerardo, Méndez-Macías / ORC ID: 0000-0001-9141-656X, CVU CONACYT ID: 645250

DOI: $10.35429 /$ JCE.2019.8.3.1.9

Recibido 03 de Marzo, 2019; Aceptado 30 Junio, 2019

\section{Resumen}

La soldadura es un proceso importante porque proporciona una unión permanente y las partes soldadas se vuelven una sola unidad. Al unir dos metales se crea una junta (unión soldada) donde tendrá ciertas características, así como también si no es aplicada de la forma correcta posiblemente quedarán discontinuidades. En cualquier proceso de soldadura se pueden presentar defectos, lo principal es asegurar que la profundidad de penetración de la soldadura sea la correcta y garantizar el óptimo desempeño de las funciones a realizar. Una empresa de Aguascalientes dedicada a la manufactura de piezas troqueladas, maquinadas y soldadas ha solicitado que se le realice un análisis a la soldadura aplicada a una pieza que la ha seccionado en 8 partes las cuales contienen los puntos de soldadura que se requiere para formar la estructura de un soporte descansabrazos. Se realizó el estudio por medio de una macro metalografía donde se expuso la principal característica que se solicitó y que es la profundidad de la soldadura. Los resultados reflejan que el proceso de soldadura está muy disperso y que requiere del control de sus parámetros. El $62 \%$ de los datos medidos cumple con lo especificado en la norma VW 01106-1 edición 2015 04 con un mínimo de $1.0 \mathrm{~mm}$ de profundidad. El detalle de estos datos se puede revisar dentro de este reporte.

Soldadura, Metalografía y Penetración de soldadura

\begin{abstract}
Welding is an important process because it provides a permanent bond and the welded parts become a single unit. When joining two metals a joint (welded joint) is created where they will have certain characteristics, as well as if it is not the application of the correct form possibly discontinuities will remain. In any welding process, defects may occur, the main thing is to ensure that the penetration depth of the weld is correct and the accuracy of the performance of the functions to be performed. A company from Aguascalientes dedicated to the manufacture of die-cut, machined and welded parts has requested that an analysis to the welding applied to a piece that has sectioned it into 8 parts which contain the welding points required to form the structure of an armrest support. The study was carried out by means of a macro metallography where the main characteristic that was requested was exposed and that is the depth of the weld. The results reflect that the welding process is very dispersed and requires control of its parameters. $62 \%$ of the measured data complies with what is specified in VW 01106-1 edition 2015 - 04 with a minimum depth of 1.0 $\mathrm{mm}$. The detail of this data can be reviewed within this report
\end{abstract}

Welding, Metallography, Welding penetration

Citación: MEDINA-MENDOZA, José Antonio, HERRERA-REYES, Naieli y MÉNDEZ-MACÍAS, Gerardo. Análisis de la soldadura aplicada en el ZSB Trager VW SUV Tiguan (soporte zsb). Revista de Ingeniería Civil. 2019. 3-8: 1-9

\footnotetext{
* Correspondencia del Autor (correo electrónico: jose.medina@utna.edu.mx)

$\dagger$ Investigador contribuyendo como primer autor.
} 


\section{Introducción}

Actualmente los procesos de soldadura en la industria metalúrgica reflejan una gran importancia y que justamente el objetivo principal es la fabricación o procesamiento de laminados, máquinas, piezas y derivados diversos, usando en todos los casos el proceso intrínseco de la soldadura, en donde son tres aspectos esenciales que se brindan a la calidad precio.

\section{- $\quad$ Una unión permanente \\ - Bajo Costo \\ - $\quad$ Seguridad y fiabilidad}

Específicamente refiriéndose al proceso de soldadura GMAW es un proceso que garantiza un cordón de soldadura libre de escoria, impurezas, continuo y uniforme. Otro aspecto importante que ofrece este proceso es la flexibilidad que permite soldar aluminio, cobre, aceros inoxidables y aceros de baja aleación en láminas a partir de $0.5 \mathrm{~mm}$ de espesor.

Una gran variedad de piezas para la industria automotriz son fabricadas con esté proceso y muy frecuentemente en chasis o armaduras de asientos y accesorios como el que se trata en este reporte técnico. Una empresa del Parque Industrial del Valle de Aguascalientes (PIVA) en la ciudad de Aguascalientes, Ags. concursa por el proyecto de la fabricación del soporte tubular soldado del descansa brazos central de un automóvil Tiguan VW, por lo cual se solicita una validación de la aplicación de soldadura en una pieza previamente cortada en sus partes principales.

La pieza es entregada en ocho partes que contienen las diferentes aplicaciones de soldadura y se proporciona también un diseño de la pieza con las características de soldadura requeridas.

El diseño indica las normatividades que hay que cumplir para la penetración de la soldadura mencionándose la norma VW 011061 edición 2015 - 04 para soldadura en atmósfera protectora.

La serie de normas VW 00106 se compone, bajo el título genérico de Soldadura en atmósfera protectora, de las partes siguientes:
Parte 1: Uniones soldadas en chapas de acero - Realización, aseguramiento de la calidad y diseño.

- $\quad$ Parte 2: Mecanizado posterior en uniones en chapas de acero.

- $\quad$ Parte 3: Uniones soldadas de aluminio.

Además, existe la VW 01142 Reparaciones en cordones de soldadura sobre estructuras de aluminio - Valoración del producto e indicaciones de procedimiento.

\section{Ámbito de aplicación}

La norma VW 00106 es válida para para la realización y el aseguramiento de la calidad de uniones soldadas por arco voltaico sobre materiales de acero, así como el diseño de dichas uniones soldadas.

Materiales: chapas de acero brillantes, sin recubrimiento y con recubrimiento, así como aceros altamente aleados y aceros inoxidables.

Geometría: Espesores de las piezas de unión de $0,5 \mathrm{~mm}$ a $10 \mathrm{~mm}$. Todas las uniones soldadas por arco voltaico, que no caigan dentro de este rango de aplicación, necesitan una aclaración con el departamento de desarrollo técnico. Se admiten indicaciones especiales condicionadas por el componente, por ejemplo, modificación del grupo de evaluación para determinadas irregularidades, y deben registrarse en el plano (en el registro de datos).

Para la aplicación de esta norma se aplican los siguientes símbolos y abreviaturas.

- $\quad \quad \quad \mathrm{t}$ Suma de los grosores de chapa, mm

- $\quad \alpha$ Ángulo de los flancos del cordón en la junta oblicua o ángulo de transición

- del cordón (irregularidad)

- a Espesor calculado de cordón angular, $\mathrm{mm}$

b Profundidad de penetración, $\mathrm{mm}$

bR Anchura del cordón de soldadura

b' Anchura del pandeo de la raíz

CE Equivalente de carbono

F Plano de la unión

- $\quad$ fi Profundidad de penetración en la chapa i, $\mathrm{mm}$

- $\quad$ fSt Afectación de la superficie (cobertura de la superficie frontal), mm o \% de la superficie frontal

h Magnitud de una irregularidad, por ejemplo, dimensión de la ranura, $\mathrm{mm}$ 


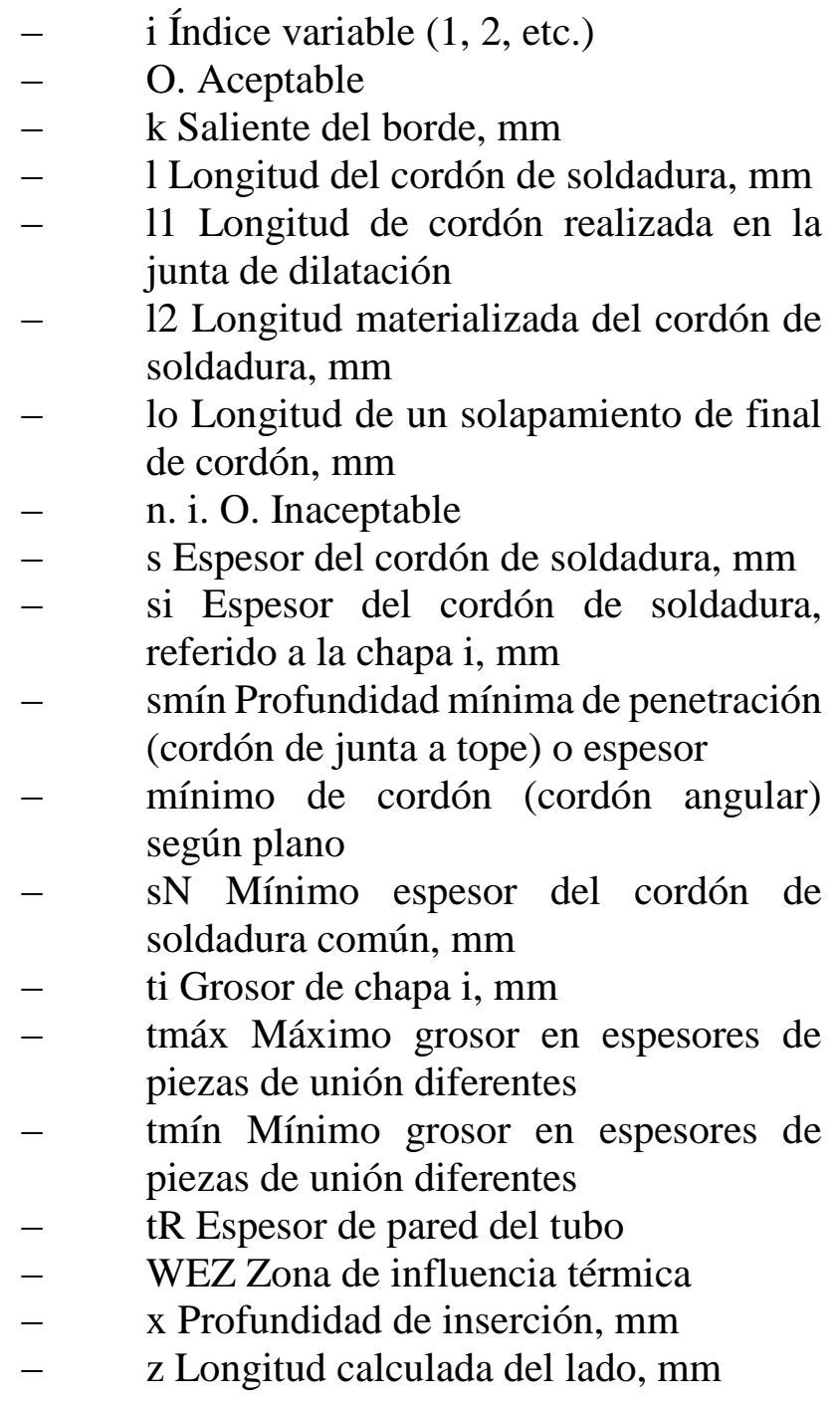

Profundidad de penetración en el caso de cordón angular en junta de solapa (soldadura de ángulo sobre borde). En el caso de que la resistencia de la chapa más fina sea notablemente mayor que la de la más gruesa, puede ser necesario un espesor de cordón mínimo mayor de smín $=0,7 \times$ tmín. En estos casos deben calcularse b1, b2 según la fórmula (1): b1, b2 $\geq 1,4 \times$ smín

\section{Justificación}

Para la empresa es necesario antes de validar de manera formal su proceso de soldadura referente al soporte ZSB, un análisis previo de las condiciones de los pilotajes de prueba desarrollados. El no cumplir con las condiciones y normatividades implica la pérdida del proyecto de fabricación. Cada zona de soldadura debe validarse para que la estructura soporte el uso para el cual está destinada y sea validada formalmente como una pieza de calidad por una instancia certificadora.

\section{Objetivo}

Validar la aplicación de soldadura en el soporte ZSB Tiguan por medio de un análisis metalográfico.

\section{Marco Teórico}

El examen de secciones materialográficas de juntas soldadas se realiza normalmente conforme a dos niveles de inspección:

Macro: Con hasta 50 aumentos empleados con estereomicroscopios. Un macroexamen normalmente se realiza en secciones transversales no embutidas en juntas soldadas e implica técnicas de corte y esmerilado fino/tosco. El acabado resultante es adecuado para el grabado, seguido de un examen de las macrocaracterísticas de la junta soldada, alguna de las cuales se cita a continuación:

- Geometría de la soldadura

- Número y tamaño de los pases

- Profundidad de penetración

- Extensión de la zona afectada térmicamente

- Imperfecciones superficiales como fracturas, mordeduras, garganta excesiva, convexidad o ángulo de soldadura.

- Imperfecciones internas como fracturas, porosidad, inclusiones metálicas, falta de fusión, falta de penetración o escoria.

- Imperfecciones en la geometría de la junta como abertura de la soldadura, cara de raíz, ángulo de chaflán o desalineación.

Micro: Cuando el examen se realiza con aumentos mayores (de hasta 1.000) con microscopios ópticos. En las técnicas de microexamen y de dureza transversal, se debe disponer de una superficie pulida y plana ópticamente. El microexamen se realiza para estudios de imperfecciones de soldadura y características microestructurales como:

- Huecos (porosidades, cavidades con contracción, fracturas diminutas)

- Fases especiales (fase-sigma en acero inoxidable)

- Tamaños/estructuras de grano

- Estructura de soldadura

- Segregación 
- Zona afectada térmicamente y estructura base

\section{Cómo realizar una inspección de soldadura}

En general, las técnicas convenciones de los ensayos de dureza y materialográficos también se aplican a las secciones soldadas. No obstante, las características de la soldadura introducen algunos retos específicos en la preparación materialográfica.

\section{Corte}

A menudo, el corte por llama se utiliza como una técnica de corte primaria para eliminar una sección soldada más manejable de una fabricación mayor. En este caso es importante que la macro/micro sección se someta a un proceso de corte abrasivo húmedo y que se seccione alejada de la influencia de daños térmicos de un proceso de corte térmico primario. A fin de minimizar la deformación del corte y el riesgo de daños térmicos en la superficie de corte, se aplican las sugerencias generales de selección del disco de corte y de los parámetros de corte.

\section{Embutición}

Normalmente, las macro secciones para los ensayos de procedimiento se preparan sin embutición debido a los límites de tiempo y porque un acabado de esmerilado fino por lo general es suficiente para un macro examen. $\mathrm{Si}$ la preparación semiautomática es una opción, entonces existen varios soportes para muestras que acomodarán las secciones transversales sin embutir de las juntas soldadas. Si se requiere la embutición, entonces existe la opción de embutición en caliente o embutición en frío. No es inusual, sin embargo, que en el examen de soldadura haya secciones transversales relativamente grandes. En este caso, se pueden utilizar los moldes rectangulares para embutición en frío.

\section{Preparación mecánica}

Lo habitual es que las secciones soldadas para un macro examen se preparen manualmente en grados cada vez más finos de papel al carburo de silicio hasta un acabado de 1200 granos. Para muestras mayores o un tiempo de esmerilado superior el disco de esmerilado de diamante en resina ofrece ventajas en cuanto a vida útil y una tasa de eliminación constante.
En el microexamen y en los ensayos de dureza transversal, los requisitos de preparación también incluyen el pulido. Las muestras de soldadura pueden conllevar múltiples variaciones en cuanto a la dureza del material en la muestra, ya sea por cambios de fase durante el soldeo o porque la junta incorpora metales diferentes. El metal de soldadura puede contener partículas duras o algún defecto propio de la soldadura. Por lo tanto, es importante que el método de preparación garantice que el relieve de pulido entre las características microestructurales sea mínimo y que se conserven todos los elementos microestructurales. En este sentido, se prefiere el equipo de preparación automático o semiautomático ya que ofrece constancia y reproducibilidad del pulido lo que facilita un análisis microestructural preciso.

\section{Ataque}

Se puede utilizar tanto el ataque químico como electrolítico, dependiendo de la aleación y el análisis requerido. El reactivo más común utilizado para el acero de baja aleación y al carbono es el nital con diversas concentraciones; también se puede usar un $10 \%$ de persulfato amónico. Para diferentes aleaciones como aceros inoxidables o aleaciones de níquel, se debe considerar el ataque electrolítico. El ataque electrolítico ofrece algunas ventajas respecto a otras soluciones de ataque químico como velocidad, facilidad de manejo y mayor seguridad ya que minimiza el contacto del usuario con los reactivos. En aplicaciones donde se requiere un análisis microestructural detallado, las muestras del pulido y ataque electrolítico deben esmerilarse hasta un acabado de 1.000 granos.

\section{Características geométricas}

Las características geométricas de una soldadura por lo general se especifican individualmente para cada soldadura o grupo de soldaduras. Por lo tanto, el análisis de fallos, la aprobación o el control de una soldadura en la mayoría de los casos se realiza con una especificación detallada. El tipo, el número y el tamaño de las imperfecciones internas o superficiales además se definen perfectamente para la calidad de soldadura. La medición, el recuento y la indicación e informe de características geométricas e imperfecciones en las soldaduras se debe al alto grado de estandarización como tarea y se puede optimizar mediante el uso de sistemas de imágenes específicos. 


\section{Metodología}

La empresa ha entregado 8 piezas cortadas donde se observan las soldaduras realizadas en el ZSB Trager y que se someten al siguiente proceso de análisis:

Metalografía Macro para observar básicamente la profundidad de la aplicación de la soldadura que es la característica de la aplicación que pide el cliente.

El estudio se desarrolló en cinco pasos:

Pulido grueso con lijas de grado 240, 600 y 1000 hasta quitar el rayado del corte previo que se les dio a las piezas en la empresa por medio de un proceso de electroerosión.

Pulido fino con lijas de grado $1500 \mathrm{y}$ 2000 que al igual que el pulido grueso se realizó en una pulidora radial marca Minitech modelo 233.

Pulido extrafino con paño y un compuesto de hidróxido de aluminio para obtener un acabado espejo en las muestras sometidas al pulido.

Ataque químico realizado con nital (alcohol y ácido nítrico) al 5\% durante 15 segundos, lavando con agua destilada y secando sin utilizar métodos de aplicación de aire.

Medición de la profundidad de la aplicación de soldadura por medio de un comparador óptico marca mitutoyo modelo Serie 303 PJ-h30a1010b.

\section{Resultados conclusiones}

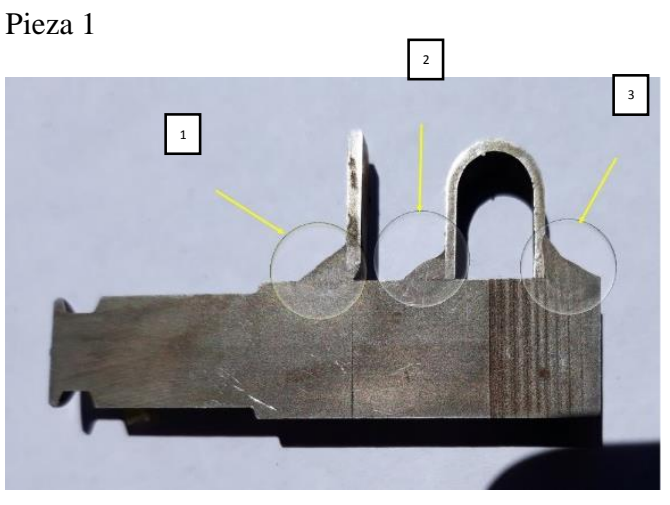

Detalle 1

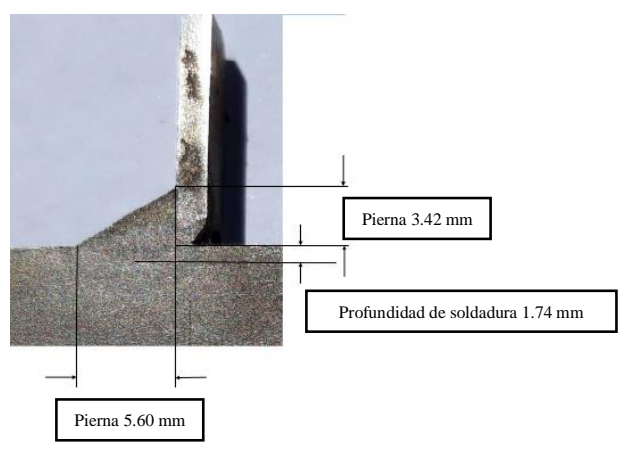

Detalle 2

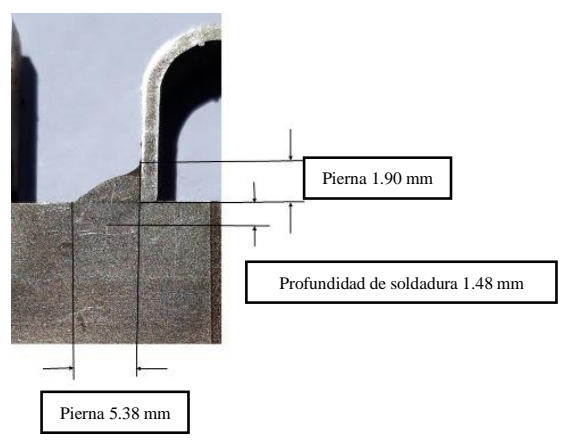

Detalle 3

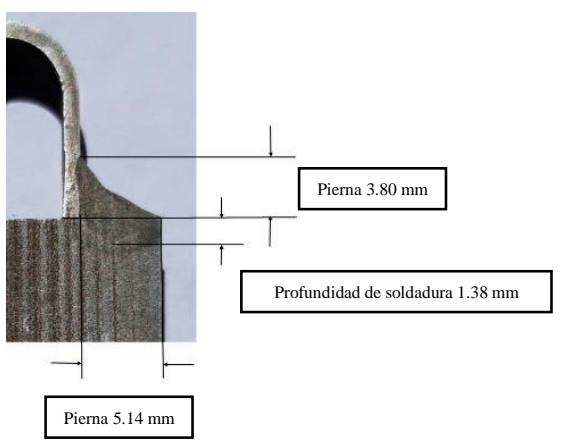

Figura 1 Detalles de Pieza 1

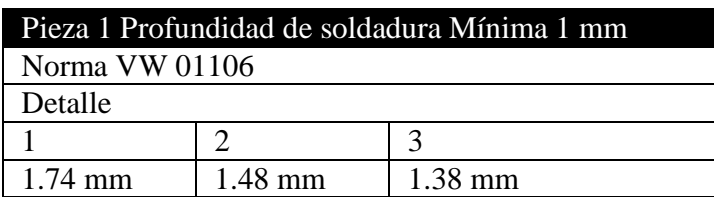


Pieza 2

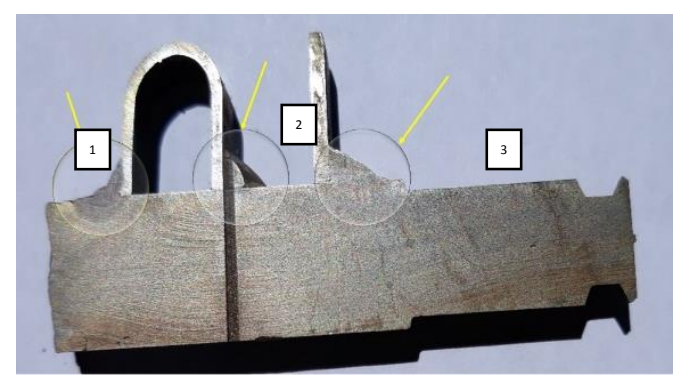

Detalle 1

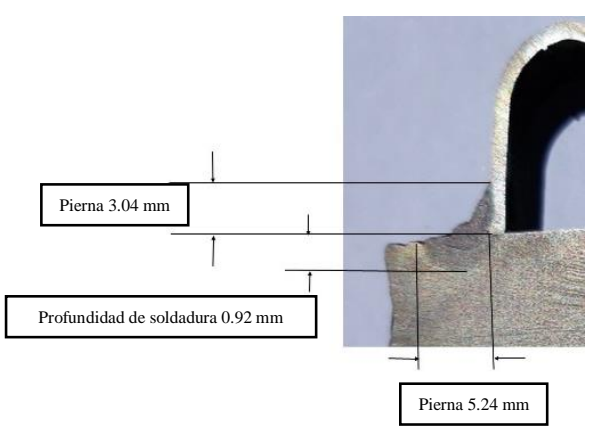

Detalle 2

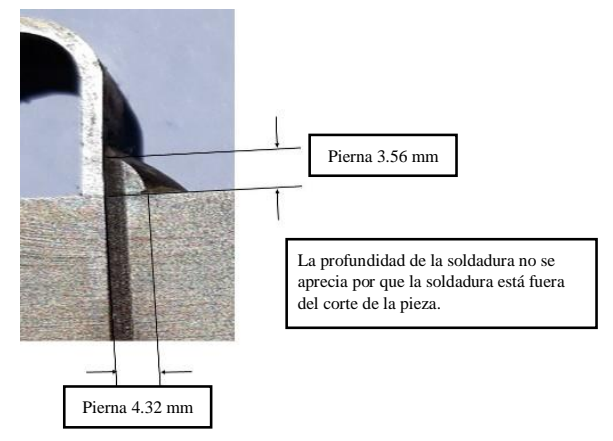

Detalle 3

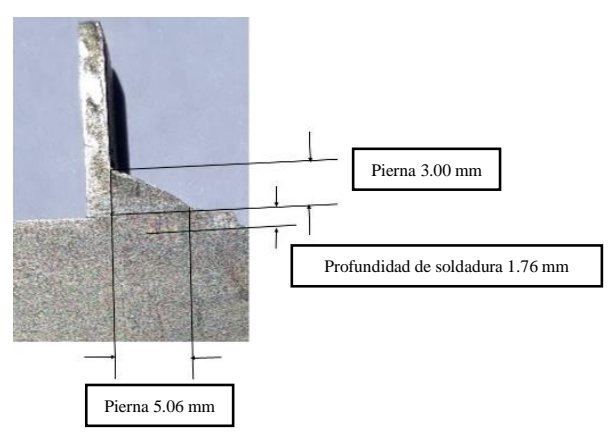

Figura 2 Pieza 2

Pieza 2 Profundidad de soldadura Mínima $1 \mathrm{~mm}$
\begin{tabular}{|l|l|l|}
\hline Norma VW 01106 \\
\hline Detalle & 2 & 3 \\
\hline 1 & N/A & $1.76 \mathrm{~mm}$ \\
\hline $0.92 \mathrm{~mm}$ &
\end{tabular}

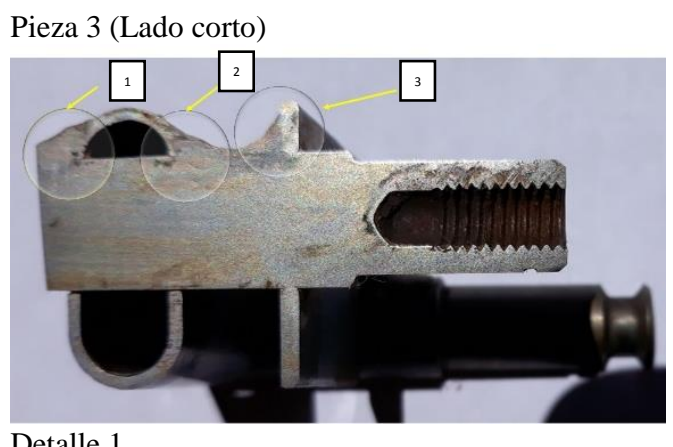

Detalle 1

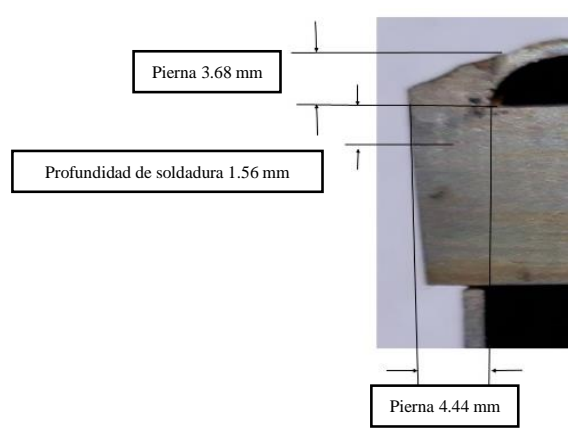

Detalle 2

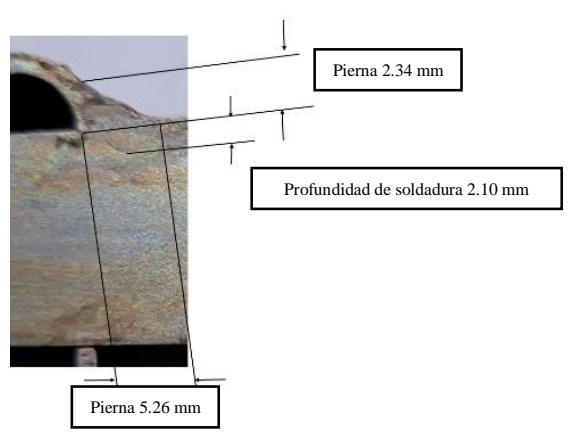

Detalle 3

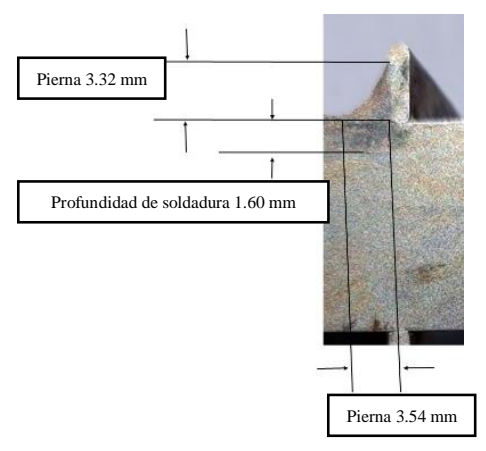

Figura 3 Pieza 3, lado corto

\begin{tabular}{|c|c|c|}
\hline Pieza & $\begin{array}{l}\text { lo corto) } \\
\text { Míni }\end{array}$ & $\begin{array}{l}\text { indidad de } \\
\mathrm{mm}\end{array}$ \\
\hline Norma VV & 106 & \\
\hline Detalle & & \\
\hline 1 & 2 & 3 \\
\hline $1.56 \mathrm{~mm}$ & $2.10 \mathrm{~mm}$ & $1.70 \mathrm{~mm}$ \\
\hline
\end{tabular}


Pieza 3 (Lado largo)

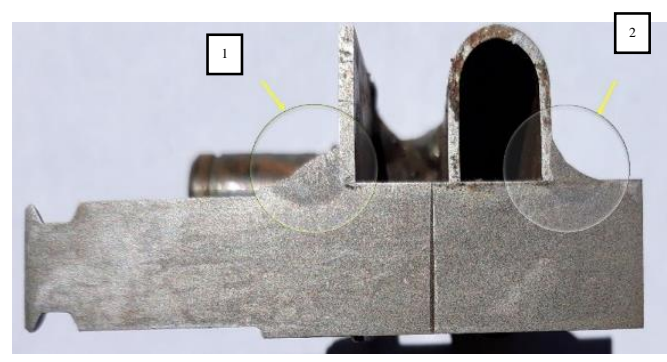

Detalle 1

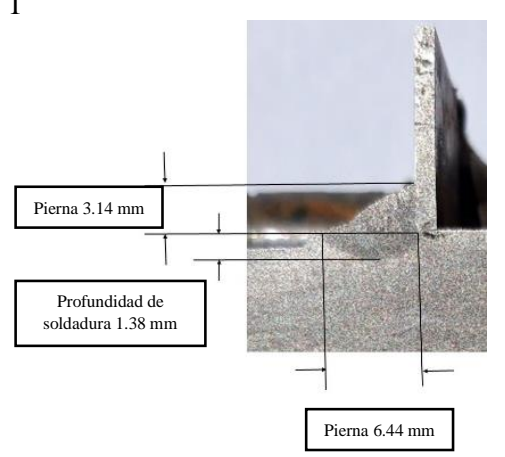

Detalle 2

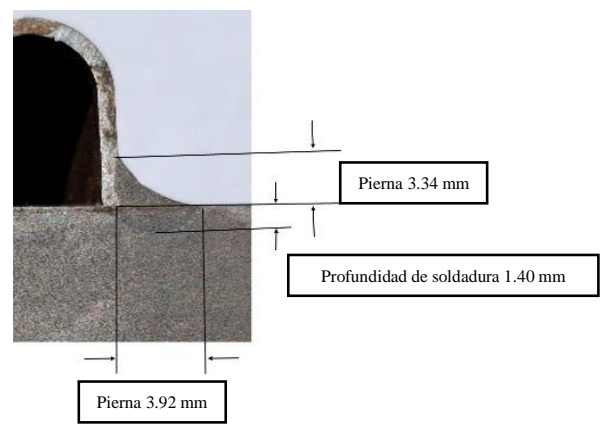

Figura 4 Pieza 3, lado largo

Pieza $3 \begin{gathered}\text { (Lado largo) Profundidad de soldadura } \\
\text { Mínima } 1 \mathrm{~mm}\end{gathered}$
\begin{tabular}{|l|l|l|}
\hline \multicolumn{3}{|c|}{ Norma VW 01106} \\
\hline Detalle \\
\hline 1 & 2 & 3 \\
\hline $1.38 \mathrm{~mm}$ & $1,40 \mathrm{~mm}$ & N/A \\
\hline
\end{tabular}

Pieza 4 (Lado largo)

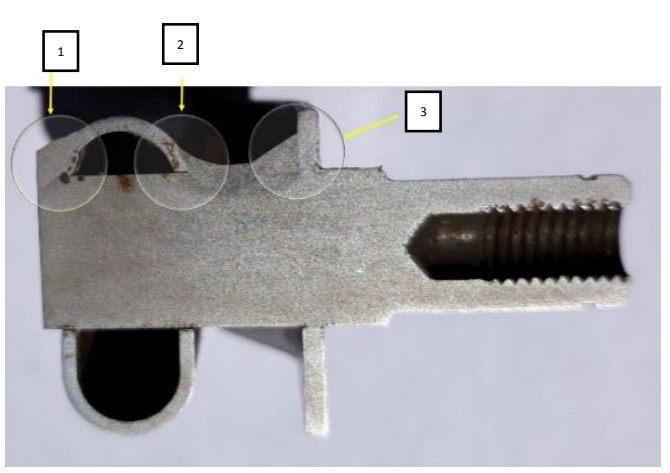

Detalle 1

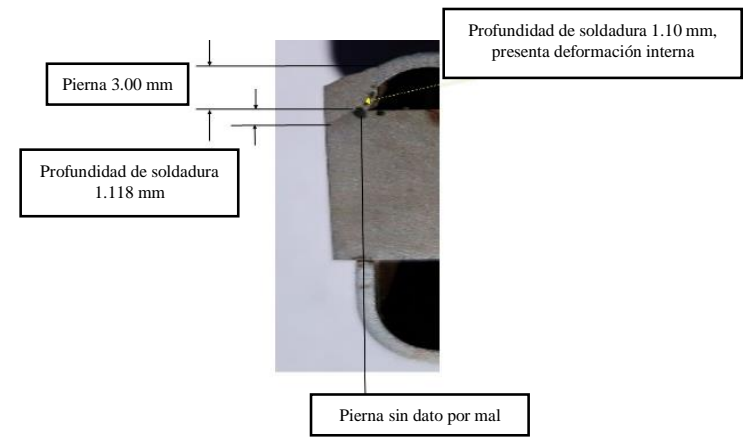

Detalle 2

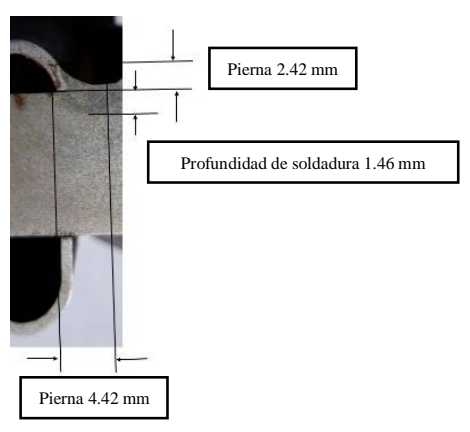

Detalle 3

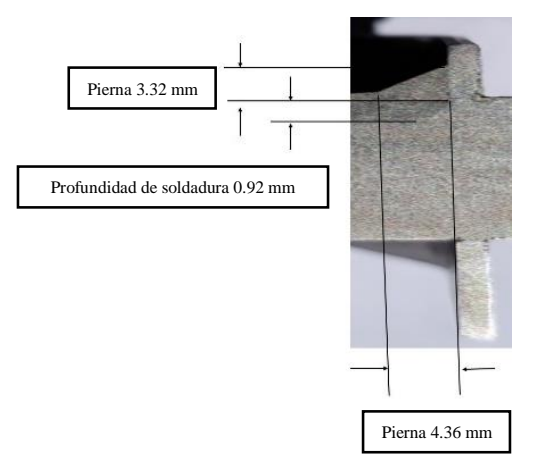

Figura 4 Pieza 4

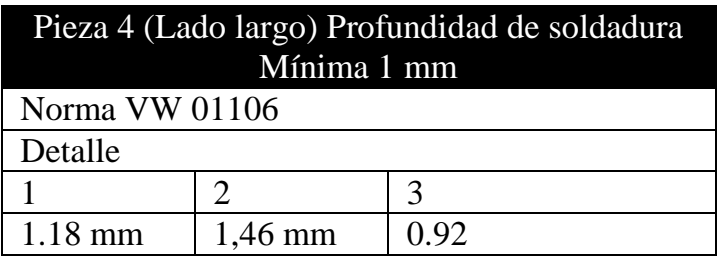


Pieza 4 (Lado corto)

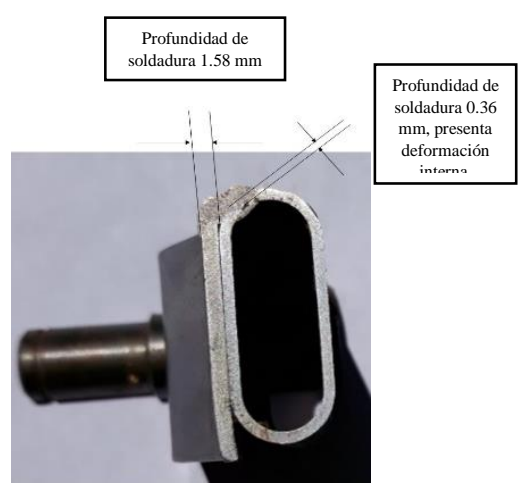

Figura 4 Pieza 4, lado corto

$\begin{array}{r}\text { Pieza } 4 \text { (Lado corto) Profundidad de soldadura } \\
\text { Mínima } 1 \mathrm{~mm} \text { y } 0.5 \mathrm{~mm}\end{array}$
\begin{tabular}{|l|l|l|}
\hline Norma VW 01106 \\
\hline Detalle & 2 & 3 \\
\hline 1 & $0.36 \mathrm{~mm}$ & N/A \\
\hline $1.58 \mathrm{~mm}$ & & \\
\hline
\end{tabular}

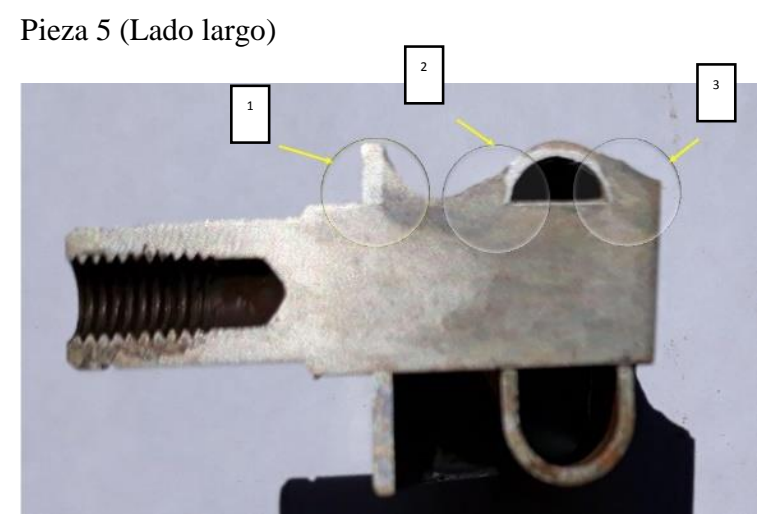

Detalle 1

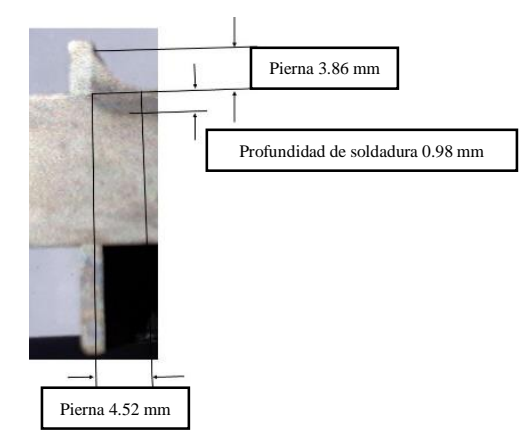

Detalle 2

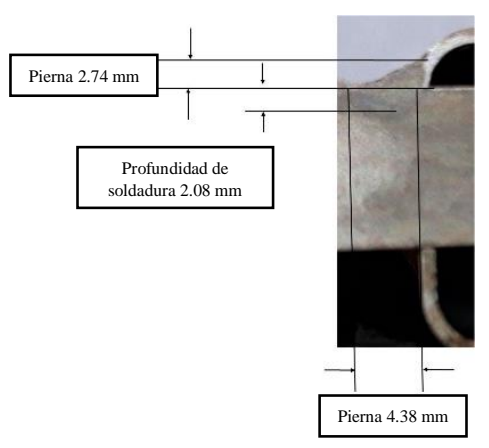

Detalle 3

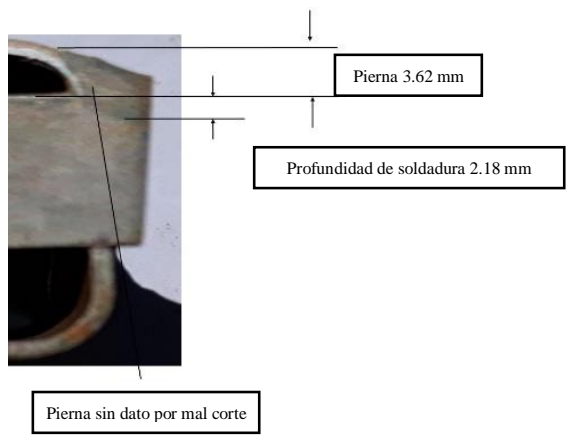

Figura 5 Pieza 5, lado largo

\begin{tabular}{|c|c|c|}
\hline \multicolumn{3}{|c|}{$\begin{array}{l}\text { Pieza } 5 \text { (Lado largo) Profundidad de } \\
\text { soldadura Mínima } 1 \mathrm{~mm} \text { y } 0.5 \mathrm{~mm}\end{array}$} \\
\hline \multicolumn{3}{|c|}{ Norma VW 01106} \\
\hline \multicolumn{3}{|l|}{ Detalle } \\
\hline 1 & 2 & 3 \\
\hline $0.96 \mathrm{~mm}$ & $2.08 \mathrm{~mm}$ & 2.18 \\
\hline
\end{tabular}

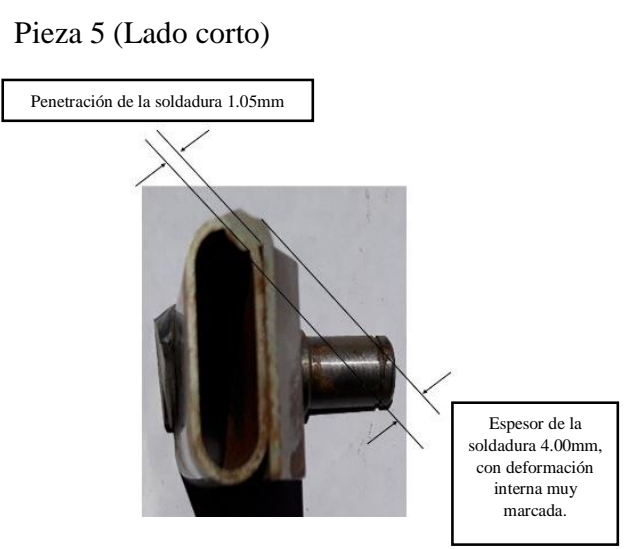

Figura 5 Pieza 5, lado corto

\begin{tabular}{|l|l|l|}
\hline \multicolumn{2}{|c|}{$\begin{array}{c}\text { Pieza } 5 \text { (Lado corto) Profundidad de } \\
\text { soldadura Mínima } 1 \text { mm y } \mathbf{0 . 5} \text { mm }\end{array}$} \\
\begin{tabular}{|l|l|}
\hline Norma VW 01106 \\
\hline Detalle
\end{tabular} \\
\hline 1 & 2 & 3 \\
\hline $1.06 \mathrm{~mm}$ & $4.00 \mathrm{~mm}$ & N/A \\
\hline
\end{tabular}

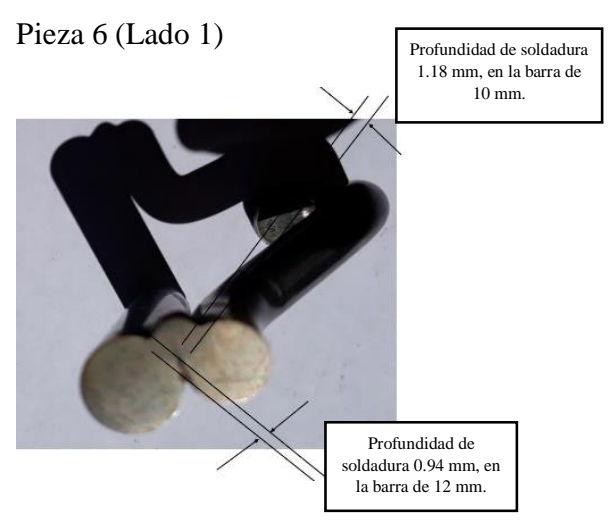

Figura 6. Pieza 6, lado 1. 


\begin{tabular}{|l}
\begin{tabular}{|l|} 
Pieza 6 (Lado 1) Profundidad de soldadura \\
Mínima 1 mm
\end{tabular} \\
\begin{tabular}{|l|l|l|}
\hline Norma VW 01106 \\
\hline Detalle \\
\hline 1 & 2 & 3 \\
\hline $1.18 \mathrm{~mm}$ & $0.96 \mathrm{~mm}$ & 2.18 \\
\hline
\end{tabular}
\end{tabular}

Pieza 6 (Lado 2)

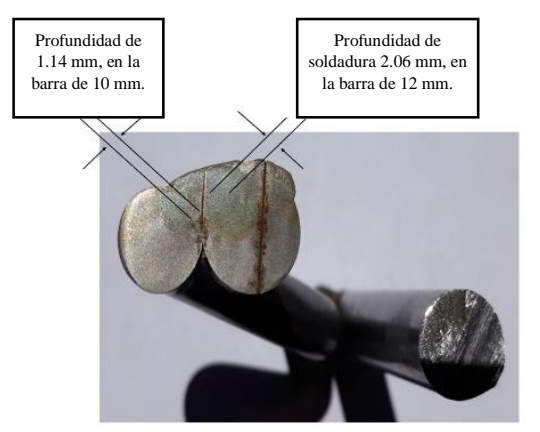

Figura 6 Pieza 6, lado 2

\begin{tabular}{|l|l|}
\hline \multicolumn{2}{|c|}{ Pieza 6 (Lado 2) Profundidad de soldadura } \\
Mínima $1 \mathrm{~mm}$
\end{tabular}

Pieza 7

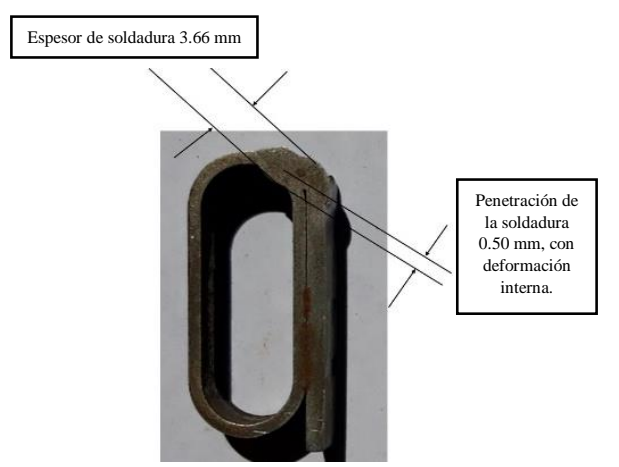

Figura 7 Pieza 7

\begin{tabular}{|c|c|c|}
\hline & & \\
\hline Norma V & 106 & \\
\hline Detalle & & \\
\hline 1 & 2 & 3 \\
\hline $3.66 \mathrm{~mm}$ & $0.50 \mathrm{~mm}$ & N/A \\
\hline
\end{tabular}

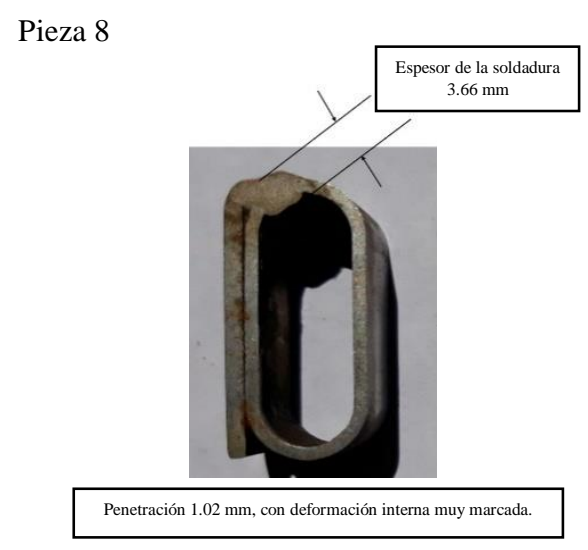

Figura 8 Pieza 8

Pieza 8 Profundidad de soldadura Mínima $1 \mathrm{~mm}$ y
$0.5 \mathrm{~mm}$
\begin{tabular}{|l|l|l|}
\hline \multicolumn{3}{|l|}{ Norma VW 01106} \\
\hline Detalle & 2 & 3 \\
\hline 1 & $1.02 \mathrm{~mm}$ & N/A \\
\hline $3.66 \mathrm{~mm}$ &
\end{tabular}

Los resultados muestran que se requiere un mejor control de la aplicación del proceso de soldadura para el ZSB Trager ya que solo el $82 \%$ de las mediciones cumplen con lo especificado y será necesario seguir observando el proceso para garantizar los resultados. El promedio de la profundidad de la soldadura es de $1.63 \mathrm{~mm}$. Es necesario cuidar los parámetros de la aplicación de la soldadura para que los resultados sean más estables. Se recomienda realizar más análisis similares durante las pruebas de pilotaje para esta pieza soldada.

\section{Referencias}

Graves, R. G. (1979). Metalografía microscópica práctica. URMO.

(2009). Norma Volkswagen VW01106. Clasificación 04817.

Rosanes, J. (2019). Proyectos de Ingeniería Metalúrgica. Editorial Bellisco.

Yu, M. L. (1977). Metalografía y tratamiento térmico de los metales. Moscú: Mir. 